\title{
Gemeinwohlvorstellungen bei Klassikern der Rechts- und Staatsphilosophie
}

\section{Methodische Vorüberlegung}

Das Thema, das ich mir gestellt habe, ist zunächst historisch orientiert. Es sollen die Gemeinwohlvorstellungen näher betrachtet und analysiert werden, die uns bei Klassikern der Rechts- und Staatsphilosophie entgegentreten - ich sage bewußt Klassikern und nicht den Klassikern, denn eine Auswahl ist für den begrenzten Rahmen unerläßlich. In welcher Weise tritt bei den zu behandelnden Klassikern der Gedanke und auch ein Begriff von Gemeinwohl auf, wie wird sein Gehalt näher bestimmt, welche Vorstellungen und Grundauffassungen über die Art und das Ziel menschlichen Zusammenlebens kommen in ihm zum Ausdruck? Lassen sich für das, was unter Gemeinwohl verstanden wird - wenn ja, in welchem Ausmaß - gemeinsame Merkmale über Zeitepochen hinweg ausmachen, oder bleibt es bei einem Auf und Ab der Verschiedenheiten, so daß Wort und Begriff nur als Formel erscheinen, eine Leerformel sozusagen, ohne zusammenfassende und steuernde geistige Kraft?

Fragen wir in dieser Weise, begeben wir uns auf das Feld der Begriffsgeschichte. Hierzu ist allerdings eine methodische Vorbemerkung erforderlich. Begriffsgeschichte steht gewiß in enger Beziehung zur Geschichte des Gebrauchs der Worte, aber sie ist nicht gleichbedeutend mit Wortgebrauchsgeschichte. 'Wäre dies der Fall, müßte unsere Untersuchung dort einsetzen, wo das Wort Gemeinwohl bzw. seine Entsprechung wie utilitas/salus publica oder bonum commune erstmals auftaucht, und es wäre der Bedeutungsgehalt und Bedeutungswandel bei den Klassikern, die es verwenden, herauszufinden. Aber das würde die Fragestellung verengen. Denn ebenso wie der Bedeutungsgehalt, den das Wort bezeichnet, sich verändern kann, vermag ein Wort nach Kontext und Verwendung auch verschiedene Bedeutungsgehalte und Begriffe zu tragen. ${ }^{2}$ Worte und die von ihnen bezeichneten Begriffe sind ja Wege, um zur Erkenntnis der Sache zu gelangen. Karl Kroeschell hat zu recht darauf hingewiesen, daß für die begriffsge-

' Siehe Koselleck 1992, S. XII: „Der Begriff haftet zwar am Wort, ist aber zugleich mehr als das Wort."

2 Vgl. Depenheuer 1988, S. 38 ff. 
schichtliche Fragestellung, wenn sie hermeneutisch reflektiert ist, das Dreieck von Wort - Sache - Begriff entscheidend ist. ${ }^{3}$ Die Sache aber, um die es bei Gemeinwohlvorstellungen und dem Gemeinwohlbegriff geht, ist die rechte Ordnung und Orientierung im und für das Zusammenleben von Menschen in (organisierten) Gemeinschaften, vorab in politischen Gemeinschaften. Diese Ordnung und Orientierung bildet den Gegenstand, auf den hin Gemeinwohlvorstellungen - welcher Art sie auch sein mögen - bezogen sind; und diese Vorstellungen finden im Gemeinwohlbegriff ihren gedanklichzusammenfassenden Niederschlag.

Unsere Frage muß daher dort einsetzen, wo solche Gedanken über Ordnung und Orientierung im und für das Zusammenleben von Menschen als reflektierte Gedanken aufkommen und begrifflich gefaßt werden, und dies unabhängig davon, ob dafür das Wort Gemeinwohl oder seine Entsprechung gebraucht wird. Die Fragestellung muß, mit anderen Worten, problemorientiert sein. Ist eine bestimmte Bezeichnung dafür, etwa Gemeinwohl, erst zu einer bestimmten Zeit auffindbar oder kommt sie von einer bestimmten Zeit an außer Gebrauch, kann dies gerade ein Indikator für bestimmte sachlich-inhaltliche Veränderungen in den zugrundeliegenden Vorstellungen sein; es bedeutet aber keineswegs, daß solche Vorstellungen - und damit das Problem - vorher nicht vorhanden waren oder seither entfallen sind.

\section{Gemeinwohlvorstellungen - von Sokrates bis Kant}

Wenden wir uns nun den Gemeinwohlvorstellungen bei etlichen Klassikern der Rechtsund Staatsphilosophie im einzelnen zu. Es kann hierbei, das gilt es noch einmal zu betonen, nicht annähernd Vollständigkeit erstrebt werden. Es ist nur möglich, markante Zeugnisse aufzugreifen, sie zu interpretieren und - abschließend - Gemeinsamkeiten und Unterschiede aufzuweisen.

1. Von Sokrates, um mit ihm zu beginnen, sind Auffassungen über die Polis und ihre gute Ordnung nicht ausdrücklich überliefert. Aber sie lassen sich aus Äußerungen, die ihm in den platonischen Dialogen zugeschrieben werden, erschließen. In Platons Politeia, die ja als Dialog über Gerechtigkeit angelegt ist, führt Sokrates ein Streitgespräch mit dem Sophisten Thrasymachos über die Gerechtigkeit und Ungerechtigkeit des Regierens und der Regierenden. Beide gebrauchen das Bild vom Hirten und der Herde, aber sie deuten es verschieden. Während Thrasymachos davon ausgeht, daß die Hirten, wenn sie die Schafe fett machen und pflegen, nur ihren Vorteil im Sinn haben, das, was gut ist für sie selbst und ihre Herren, ${ }^{4}$ stellt Sokrates ihm die wahre Hirtenkunst gegenüber. Diese besteht nicht darin, die Schafe für einen guten Schmaus zu mästen, wie ein Feinschmecker, oder um einen Verkaufsgewinn zu erreichen, wie ein Erwerbsmann. Sie hat vielmehr ,nur die eine Aufgabe, für das ihr anvertraute Gut, worüber sie gesetzt ist, fürs beste zu sorgen".5 Und so müsse man sich eingestehen, hält er Thrasymachos ent-

3 Vgl. Kroeschell 1983.

4 Platon, Politeia, 343 b-c.

5 Ebd., $345 \mathrm{c}-\mathrm{d}$. 
gegen, „daß jede Regierung, sofern sie Regierung ist, das Beste für nichts anderes als für das Beherrschte und Betreute bedenke"."

In dieser Deutung des Hirtenbildes geschieht gegenüber der eher selbstnützigen Position der Sophisten etwas Weittragendes: Herrschaft und Regierung werden statt am Ruhm und Machtinteresse der Herrscher an der Sorge für das Beste der Regierten, ihr Gemeinwohl, orientiert und damit an Fremdnützigkeit. Diese wird zu deren Kriterium, und es öffnet sich der Blick für den Amtsgedanken als Grund und Telos von Herrschaft. Allerdings ist dieser Gemeinwohlvorstellung mit dem Bild von Hirt und Herde durchaus auch ein autoritativer Zug eigen. Die politische Gemeinschaft erscheint nicht als Vereinigung mündiger Bürger, die einen der ihren mit der Regierung beauftragen; das Wohl der Regierten wird vielmehr besorgt vom - ihnen überlegenen - Hirten, von ihm werden sie geleitet, sind Objekte seiner Fürsorge. ${ }^{7}$ Das entspricht der Auffassung vom Charakter der Polis, die Sokrates hatte. Sie wird von ihm verstanden, wie sich im Dialog Kriton zeigt, als die alle einzelnen umfangende Ordnung, die höher steht als der einzelne; das Verhältnis des einzelnen zur Polis ist dem eines Kindes zu seinen Eltern vergleichbar. ${ }^{8}$

2. Platon entwickelt in seiner Politeia ${ }^{9}$ auf der Suche nach Gerechtigkeit eine ideale Polisordnung, die Ausdruck von Gerechtigkeit ist. Gerechtigkeit meint für Platon einen Zustand der Wohlgeordnetheit, der Verschiedenes zur Einheit führt und darin erhält. ${ }^{10}$ Sie hat ihr Anwendungsfeld zum einen im einzelnen Menschen - hier besteht sie in der angemessenen, harmonischen Zuordnung der verschiedenen Seelenkräfte ${ }^{11}-$ zum andern in der Polis. In der Polis besteht sie in der Harmonie bei der Wahrnehmung der Grundfunktionen durch die dazu jeweils befähigten und berufenen Glieder der Polis, die einzelnen Menschen, die jeweils das Ihrige, was sie vermögen, beitragen und dabei bleiben und eben dadurch eine Wohlgeordnetheit der Polis bewirken.

Wie sieht diese Vorstellung von der guten, die Gerechtigkeit verwirklichenden Ordnung der Polis des Näheren aus? Sie nimmt ihren Ausgang - aller Polemik gegen den ,totalitären" Platon zum Trotz - vom einzelnen Menschen und seinem vollendeten Leben, ihr Ziel sind nicht kollektive oder transpersonale Güter. Aber sie ist in ihrem Umwillen nicht auf den Bereich der äußeren Bedürfnisnatur der Menschen beschränkt, auch die geistig-kulturelle und ethisch-sittliche Entfaltung können die einzelnen nicht je für sich, sondern erst in der und durch die Polis erreichen. ${ }^{12}$ Die Einbeziehung der ein-

${ }^{6}$ Ebd., 345 d.

7 In dieser Ausdeutung wirkt das Bild vom Hirt und den Schafen auch im Neuen Testament, vgl. Joh. 10, 1-18; Hebr. 13,20.

8 Platon, Kriton, 50 d-e.

9 Im Folgenden beschränke ich mich auf Platons Politeia und lasse den Politicos sowie die Nomoi außer Betracht.

10 Platon, Politeia, 441 d-e.

11 Sie wird dadurch hergestellt, daß der Vernunft das Herrschen zukommt, da sie weise ist und die Obsorge für die ganze Seele hat, dem Mutbegehren aber, daß es der Vernunft gehorsam und ihr Helfer ist und daß beide das Begehren beherrschen, das den größten Teil der Seele ausmacht und von Natur aus am unersättlichsten ist, vgl. Platon, Politeia, 441 e-442 a.

12 Dies ist der sachliche Gehalt des Gründungsmythos der Polis, wie er in der Politeia, 369-382 vorgestellt wird. 
zelnen in die Polis und ihre Ordnung ist umfassend, erstreckt sich auf die Lebensbereiche und Lebensäußerungen insgesamt.

Die gerechte Ordnung der Polis, das gemeine Wohl, das sich in ihr verwirklichen soll, hat nun bei Platon primär einen institutionell-funktionsbezogenen Charakter, erst sekundär enthält es ethisch-normative Anforderungen. Platon geht davon aus, daß die Menschen nach natürlicher Anlage und erworbener Befähigung unterschiedlich sind und sich dies typenmäßig ausprägt. ${ }^{13}$ Auf dieser Grundlage wird dann die Polisgemeinschaft keineswegs etwa nach dem Geschlechterprinzip oder nach Familienbindungen, auch nicht geburtsständisch gegliedert, sondern nach den in der Polis zu realisierenden Grundfunktionen, die zueinander - entsprechend den einzelnen Seelenkräften - in einer hierarchischen Bezogenheit stehen: Die Leitung der Polis muß nach Vernunft und Einsicht erfolgen, die Sicherung nach außen - Selbstbehauptung im Kampf - durch eigens dafür Befähigte und Ausgebildete, die Sorge für die materiellen Lebensbedürfnisse wie auch für Spiele, Kunst und Kultur durch die zahlreich dafür Tätigen je nach ihrem Können. Diese Aufgaben und Funktionen stehen nicht im Konflikt miteinander, sie ergänzen sich; unter der Vorrangstellung von Vernunft und Einsicht in der Hand der Regierenden können sie die ausgeglichene Harmonie der Polisordnung hervorbringen.

Hiernach liegt die Gerechtigkeit der Polisordnung, das Gemeinwohl der Polis, zunächst einmal im Ausschluß aller Unbeteiligtheit an der Polisgemeinschaft. Jeder, der der Polis zugehört, hat einen spezifischen Anteil am Polisgeschehen, und dieser Anteil bestimmt sich nicht nach Privilegien oder Reichtum, sondern nach seiner Naturanlage und seinen ausgebildeten Fähigkeiten. Unter der Voraussetzung natürlicher Unterschiedlichkeit der Menschen herrscht ein streng sachbezogenes, nicht ein willkürliches Prinzip. Jeder ist in der Weise beteiligt, daß er das, was er vermag (das Seinige), zum Polisleben beiträgt und dabei bleibt - hieran zeigen sich (sekundär) ethisch-sittliche Anforderungen an die einzelnen; Über- und Unterordnung leiten sich nicht aus Vererbung oder Tradition her, sondern allein aus Aufgabe und Funktion.

Es macht die Eigenart dieser Gemeinwohlvorstellung aus, daß der einzelne voll in der und für die politische Gemeinschaft als deren Teil und Glied lebt: Der Gedanke einer polisfreien Individualsphäre tritt nirgends hervor. Das zeigt sich besonders bei den Wächtern und den aus ihnen zu entnehmenden Herrschern bzw. Regierenden. Sie werden einem umfassenden Erziehungsprogramm unterworfen, ${ }^{14}$ das auch - mit für uns absonderlichen, totalitär anmutenden Konsequenzen - die Lebensführung ergreift. Erreicht werden soll, daß die Wächter ganz für ihre öffentliche Funktion leben, losgelöst von familiär-privaten Bindungen und jeglichen partikularen Interessen, weil nur so dasjenige, was der Polisgemeinschaft als ganzer und ihrer vernunftgemäßen Ordnung dient, gesehen und verwirklicht werden kann. ${ }^{15}$ Die Gemeinwohlvorstellung, die Platon für seine ideale Polisordnung zugrundelegt und entfaltet, ist eine solche, in der die selbstbestimmte individuelle Freiheit in einem dem Zugriff der Polis entzogenen Be-

\footnotetext{
13 Vgl. dazu Graeser 1994, S. 184 f.

${ }^{4}$ Im einzelnen dazu Guthrie 1975, S. 449-460, 521-527.

15 Eine eingehende Interpretation dieser Anforderung gibt Gigon 1976, S. 373-419.
} 
reich keinen Ort hat. Wie Hegel es gesagt hat: „Sie [die platonische Republik] hat dies Wesentliche, daß das Prinzip der Einzelnheit unterdrückt ist. “16

3. Die Gemeinwohlvorstellung, die bei Aristoteles wirksam ist, tritt an zwei Stellen seiner Politik deutlich hervor, zum einen in seiner Darlegung des telos der Polis, zum andern in der Lehre von den Verfassungsformen und der Frage nach der besten Verfassung. Sie ist von dem Bestreben getragen, zur Stabilisierung der Polis, die nach der Krise Ende des 5. Anfang des 4. Jahrhunderts v. Chr. in Gang gekommen war, weiter beizutragen.

a) Das telos der Polis, ihr eigentliches Um-willen, ist für Aristoteles das gute, tugendhafte Leben, die eudaimonia. Zwar ist die Polis um des äußeren Lebens und seiner Sicherung willen entstanden, aber sie besteht um des guten, vollendeten Lebens willen. ${ }^{17}$ Sie hat daher ethisch-sittlichen Charakter, ist ein (auch) ethisches Gemeinwesen, dessen Zweck und Ziel über die Lebenssicherung und die Befriedigung äußerer Bedürfnisse weit hinausgeht. Eine Polis ist, wie Aristoteles sagt, ,,sowohl für die Häuser, wie für die Geschlechter die Gemeinschaft um einer vollkommenen und autarken (sich selbst genügenden) Lebensweise willen". ${ }^{18}$ Diese Lebensweise ist nicht von den einzelnen Bürgern und ihrem guten vollendeten Leben abgelöst, sie besteht gerade darin, dieses Leben der einzelnen Bürger, das ein Leben in der Polisgemeinschaft ist, zu ermöglichen. Die Glückseligkeit des einzelnen wie der Polis ist für Aristoteles dieselbe. ${ }^{19}$ Allerdings gilt dies, das darf nicht übersehen werden, nur für die Bürger der Polis, nicht für alle Menschen, die in der Polis leben. Metöken und Sklaven sind keine Bürger, der Bürgerbegriff des Aristoteles ist ein Statusbegriff. ${ }^{20}$

Die Polis umgreift auch das geistige, ethische, künstlerische, religiös-kultische Leben, kurz alle Bereiche, in und an denen sich ein vollendetes Leben als gemeinsames Leben der einzelnen entfaltet. Entsprechend umfassend ist das gemeinsame, gemeinschaftliche Gut, es hat ebenfalls ethisch-sittlichen Charakter. Die Erziehung der Bürger spielt darin eine wichtige Rolle. Die Poliskunst (als die Leitung der Polis), sagt Aristoteles in der Nikomachischen Ethik, kümmert sich am meisten darum, die Bürger in den Besitz gewisser Qualitäten zu setzen, sie zur Tugend zu bilden und fähig, das Edle zu tun. ${ }^{21}$ Das gewinnt Gestalt durch die Bildung der Jugend und zahlreiche, weite Bereiche der Lebensführung betreffende nomoi; Aristoteles unterscheidet sich von Platon insoweit nicht prinzipiell, wohl aber graduell. ${ }^{22}$

${ }^{16}$ Hegel 1959b, S. 293.

17 Aristoteles, Politik I, 2, $1252^{\mathrm{b}}, 28$.

${ }^{18}$ Ders., Politik III, 9, 1280 ${ }^{\mathrm{b}}$, 33-34, (Übersetzung E.-W. B.).

19 Vgl. ders., Politik VII, 1-2, $1323^{\mathrm{b}} 39-1324^{\mathrm{a}} 6$.

${ }^{20}$ Vgl. ders., Politik III, $1275^{\mathrm{a}}, 7-8,22-23$ : „Der Bürger aber ist nicht Bürger aufgrund der Tatsache, daß er irgendwo wohnt (denn sowohl Metöken als auch die Sklaven haben gemeinschaftlichen Anteil am Wohnen). [...] Ein Bürger schlechthin wird durch nichts anderes besser definiert als durch die Teilhabe an Rechtsprechung und Herrschaft." (Übersetzung E.-W. B.) Vgl. ferner Politik III, 5, $1278^{\mathrm{a}}, 8-12$.

21 Vgl. Aristoteles, Nikomachische Ethik I, 10, $1099^{\mathrm{b}}, 30 \mathrm{f}$.

22 Siehe dazu die Erörterungen bei Aristoteles, Politik VII, 14, die sich auf Unterricht und Gewöhnung in allen Tugenden, nicht zuletzt durch die Anordnungen des Gesetzgebers, beziehen, ferner die Empfehlungen (Politik VII, 16) für gesetzliche Vorschriften über das Alter und die - durch ent- 
Kommt nun die Polisgemeinschaft an ihr Ziel, ist auch die gerichtete Bewegung des sozialen und politischen menschlichen Seins an ihrem Ziel. Sie enthält dann in sich alle Bedingungen menschlicher Lebensbewältigung und eines vollen menschlichen Seinkönnens (allerdings nur für die Bürger), zu dem auch die Muße und die Möglichkeit gehört, sich mit theoria zu beschäftigen; die eudaimonia als das Gemeinwohl der Polis ist perfekt. Deshalb erscheint sie als (potentiell) vollkommene, sich selbst genügende Gemeinschaft - societas perfecta.

b) Aristoteles unterscheidet, um auf das Institutionelle überzugehen, die Regierungsoder Verfassungsformen in zweifacher Weise, einmal nach der äußeren Organisationsform, d. h. danach, wer die Regierung innehat, zum andern danach, zu welchem Zweck die Regierung ausgeübt wird: Zielt die Regierung auf das gemeine Beste oder zielt sie auf den eigenen Vorteil der Regierenden? Je nachdem handelt es sich um eine gute, das telos der politischen Gemeinschaft und damit das Gerechte verwirklichende, oder um eine schlechte, entartete Verfassungsform. ${ }^{23}$

Die gute, das gemeinsame Beste realisierende Regierungsform wird so über den Telosgedanken mit der Gemeinwohlvorstellung verbunden, wie sie im natürlichen Ziel und der guten Ordnung der Polisgemeinschaft Ausdruck gefunden hat. Aber Aristoteles entwickelt dies nun nicht im einzelnen nach Art eines ethisch-normativen Konzepts, vielmehr richtet er sein Augenmerk auf die institutionellen Bedingungen, die dazu führen, daß das gemeine Beste erreicht wird. Die Frage nach der Verwirklichung des Gemeinwohls mutiert zur Frage nach der besten Verfassung der Polis. Dies aber nicht im Sinne einer Idealverfassung, wie bei Platon, sondern im Sinne der relativ besten Verfassung, die so gestaltet ist, daß sich durch ihre institutionellen Vorkehrungen unter den gegebenen Bedingungen das gemeine Beste voraussichtlich einstellt. ${ }^{24}$ Diese das Gemeinwohl hervorbringende Verfassung ist für Aristoteles die gemischte Verfassung, die sich als ein Mittleres zwischen verschiedenen Extremen darstellt und am ehesten vor einem Umschlag in die Entartung sichert. ${ }^{25}$ Die Demokratie kann das für Aristoteles nicht sein - er zählt sie zu den Entartungsformen ${ }^{26}$ - denn sie dient ihrer Struktur nach dem Nutzen der Armen wie die Oligarchie dem Nutzen der Reichen, nicht aber dem gemeinen Besten. ${ }^{27}$

Die Eigentümlichkeit der gemischten Verfassung und damit das Gemeinwohl der Polis liegt für Aristoteles - man höre und staune - in der Stärke und Herrschaft des mittle-

sprechende Erziehung herbeizuführende - Leibesbeschaffenheit der Ehebewerber, das Verhalten der Frauen während der Schwangerschaft, die Aussetzung verkrüppelter Kinder, die Strafe für Ehebruch u. a. m.

$23 \mathrm{Vgl}$. Aristoteles, Politik III, 6, 1279, $17-22$.

24 Vgl. ders., Politik IV, 11, $1295^{\mathrm{a}}, 25-30$.

25 Vgl. ders., Politik IV, 11, 1295, $30-35$.

${ }^{26}$ Nach Schütrumpf 1991, S. 468 ff. war die Zuordnung der Demokratie zu den entarteten Polisformen in der damaligen Zeit nicht allgemein; sie sei von Aristoteles unter dem Gesichtspunkt der Verbindung sozialer Interessenlagen mit dem Qualifikationsmerkmal Eigennutz getroffen. Kamp 1985, S. 269 ff. ist der Auffassung, daß für Aristoteles die Demokratie wegen ihres formalen Freiheits- und Gleichheitsbegriffs das politisch Gerechte verfehle.

27 Vgl. Aristoteles, Politik III, 7, 1279, $6-10$. 
ren Besitzes. Dies ist der - institutionelle - nomos der Polis. Sein Argument: Der mittlere Besitz gehorcht am leichtesten der Vernunft; dem übermäßig Schönen, Starken, Reichen, auch dem Mann von edler Geburt fällt dies ebenso schwer wie dem übermäßig Armen, Schwachen oder Verachteten. Die ersteren haben weder Neigung noch Einsicht, anderen zu gehorchen, weil sie es von Jugend auf nicht gelernt haben, während die letzteren allzu unterwürfig sind. So können die einen nicht herrschen und nur in sklavischer Weise gehorchen, die andern nicht einer Herrschaft gehorchen, sondern nur in despotischer Weise selbst herrschen. Dann aber gibt es keine Polis von Freien, die im Herrschen und Gehorchen einander gleich sind. ${ }^{28}$ So besteht das größte Glück für die Polis und ihr Gemeinwohl darin, wenn die, welche die Herrschaftsaufgaben wahrnehmen, ein mittleres und ausreichendes Vermögen besitzen. ${ }^{29}$

4. Wenden wir uns dem Römer Cicero zu. Bei ihm tritt im Blick auf das Gemeinwohl die Berufung auf die utilitas communis bzw. publica, den gemeinsamen öffentlichen Nutzen, deutlich hervor. Dieser bezieht sich, dem auf Praxis gerichteten politischen Denken der Römer entsprechend, primär auf den Bestand und die Festigung der römischen Res publica und ihrer Ordnung. ${ }^{30}$ Aber es wäre falsch, daraus auf ein reines Nützlichkeitsdenken - etwa eine Vorform moderner Staatsräson oder des philosophischen Utilitarismus - zu schließen. Auch für Cicero geht es bei der Frage nach dem öffentlichen Nutzen und der Ordnung der Res publica um eine Frage der Gerechtigkeit - Gerechtigkeit ist für ihn, insoweit an Platon anknüpfend, Grundlage und Formprinzip auch für die politische Ordnung. ${ }^{31}$ Nur erhält diese Frage für den Römer, der am politischen Leben in den magistralen Ämtern und als Redner direkt beteiligt ist, stärker auf die gegebene politische Wirklichkeit ausgerichtete Züge.

Betrachten wir die berühmt gewordene Definition der Res publica; sie hat nicht lediglich beschreibenden, Wirklichkeit abbildenden Charakter, enthält vielmehr zugleich normative und legitimatorische Momente in sich:

„Est igitur ... res publica res populi, populus autem non omnis hominum coetus quoquo modo congregatus, sed coetus multitudinis iuris consensu et utilitatis communione sociatus. ${ }^{332}$

28 Siehe im einzelnen ders., Politik IV, 11, 1295 $1-22$.

${ }^{29}$ Vgl. ders., Politik IV $, 11,1295^{\mathrm{b}} 40-41$. Die richtige Güterverteilung als Verfassungs- und Gemeinwohlfrage war in den frühen, durch Landbesitz strukturierten Gesellschaften vielfach bewußt. Für Israel sah das Gesetz vor, daß in jedem 49. Jahr (Jubeljahr) die ursprünglich bestehende Landverteilung wieder herzustellen sei, zwischenzeitliche Besitzübertragungen im Jubeljahr wieder rückgängig zu machen waren, mit einer Ausnahme für städtische Wohngrundstücke (Leviticus 25, 9-31). Allgemein zum Problem: Schmitt 1958.

${ }^{30}$ Siehe Meyer 1962, S. 235-274.

31 Das dritte Buch von De republica ist als Dialog um Gerechtigkeit und ihre Notwendigkeit für Bestand und Ordnung der Res publica angelegt; im ersten Buch wird die Frage nach der Regierungsform als Frage nach der von Natur richtigen und besten, d. h. der gerechten Regierungsform diskutiert.

32 Cicero, De republica 1, 25, 39: „Es ist also die Res publica die Sache des Volkes; ein Volk aber ist nicht jede irgendwie zusammengebrachte Vereinigung von Menschen, sondern die Vereinigung einer Menschenmenge, verbunden durch die Anerkennung des Rechts und die Gemeinsamkeit des Nutzens." (Übersetzung E.-W. B.) 
Die Res publica erscheint als Sache des Volkes; sie wird damit abgegrenzt von einer Sache des Herrschers und einer Sache der einzelnen; der Bezug auf Gemeinsamkeit und ein Allgemeines ist ihr immanent. Sie wird als Gemeinwesen begriffen, und was dieses Gemeinwesen trägt und lebendig erhält - in der näheren Bestimmung des Volkes kommt es zum Ausdruck -, ist ebenso die Verwirklichung des gemeinsamen Nutzens wie die Anerkennung und Verwirklichung des Rechts. Das klingt und ist nüchterner und erdverbundener als die eudaimonia, das vollendete tugendhafte Leben des Aristoteles, aber es hat durchaus eigenen ethischen Bezug. Die Aussage über den consensus iuris muß nämlich vor dem Hintergrund des von Cicero entwickelten Begriffs des Rechts gesehen werden. ${ }^{33}$ Consensus iuris meint eine Übereinstimmung in der Anerkennung von Recht im Sinne des von Natur Rechten, durch die das Volk - der coetus multitudinis - abgehoben wird von einer bloßen Menge oder Masse. Keineswegs ist also jede empirische politische Ordnung bereits eine Res publica, sie ist es nur dann, wenn die erwähnten gemeinsamen Güter, die ihr Gemeinwohl ausmachen, realisiert sind.

Damit dies nun geschieht, muß für die Ordnung der Res publica eine Führungsgewalt (imperium) bestehen - der Römer Cicero sieht sie in den Magistraten -, die das Richtige, Nützliche, mit den Gesetzen und dem Recht Übereinstimmende vorschreibt, sowie ein Gefüge miteinander verknüpfter Institutionen. Cicero wendet sich, wie schon Platon und Aristoteles, einer institutionellen Betrachtung des gemeinen Wohls und Nutzens zu. Was ist die richtige, beste Verfassung der Res publica, damit ihr gemeinsames Gut auch erreicht wird und sich verwirklicht? Cicero tritt - wie Aristoteles - für eine gemischte Verfassung ein, die Elemente der drei Regierungsformen verbindet. Aber die Begründung ist gegenüber Aristoteles weniger pragmatisch, sie ist prinzipieller und weist auf die ethische Dimension zurück. Der Vorzug für die gemischte Verfassung ergibt sich nämlich aus der Notwendigkeit einer ausgeglichenen Verteilung von Recht, Pflicht und Leistung im politischen Gemeinwesen; nur dann kann die Verfassung Beständigkeit und eine Form auf Dauer haben. Bezogen auf die Verfassung Roms sagt Cicero:

„Wenn es in der civitas nicht diese ausgeglichene Gegenseitigkeit von Recht, Pflicht und Leistung gibt, so daß ausreichend Macht bei den Magistraten liegt, ausreichend Einfluß beim Rat der führenden Männer und ausreichend Freiheit beim Volk, kann dieser stabile Zustand der Res publica nicht aufrecht erhalten werden. ${ }^{\text {(34 }}$

Eine solche ausgeglichene Verteilung von Recht, Pflicht und Leistung begründet unterschiedliche Macht- und Teilhabepositionen, balanciert sie zugleich auch, und sie verpflichtet durch Teilhabe zur Verantwortung. Cicero bindet das ganze Konzept auch in eine Argumentation von der Gerechtigkeit und Gemeinschaftsbindung der Menschen her ein. In De officiis sagt er:

„Da wir aber, wie zutreffend von Platon beschrieben worden ist, nicht nur für uns selbst geboren sind, sondern einen Teil unseres Daseins die Vaterstadt beansprucht, einen Teil die Freunde, und - wie es die Stoiker meinen - was auf Erden hervorgebracht wird, insgesamt zum Nutzen der Menschen geschaffen wird, die Menschen aber um ihrer Mitmenschen willen gezeugt sind, damit sie, einer dem andern, von sich aus sich gegenseitig nützen können, so müssen wir

${ }^{33}$ Siehe ders., De legibus I, 15, 42 und De republica III, 22, 33 sowie Girardet 1983, S. 65-75.

${ }^{34}$ Cicero, De republica II, 33, 57. (Übersetzung E.-W. B.) 
darin der Natur als Führerin folgen, den gemeinsamen Nutzen - communis utilitates - in den Mittelpunkt stellen, durch Ausgleich der Pflichten - durch Geben und Nehmen - durch Fertigkeiten, Opferbereitschaft und [finanzielle] Mittel unter den Menschen das Band menschlicher Zusammengehörigkeit festigen. ${ }^{\text {. } 35}$

Ciceros Gemeinwohlvorstellungen weisen auch über die Res publica hinaus. Die gegliederte Hierarchie des Gemeinschaftslebens der Menschen schließt für ihn nicht mit der Polis oder der Res publica ab, sie greift aus in die societas generis humanis, die Gemeinschaft aller Menschen untereinander. Deren Band ist die gemeinsame Vernunft der Menschen und ihr Sprachvermögen; beide verbinden und versöhnen die Menschen in einem natürlichen Gemeinschaftsgeist durch ihr Lehren und Lernen, durch Gespräche mit- und gegeneinander, durch ihr Urteilen. Diese Verbindung hat auch eine eigene Ordnung, ein eigenes gemeinsames Gut. „In ihr ist“, sagt Cicero, „der gemeinschaftliche Umgang mit allem, was die Natur zum gemeinsamen Nutzen der Menschen hervorgebracht hat, zu bewahren ${ }^{\text {‘36 }}$ - Ansatzpunkt für eine weltweite Ordnung der Nutzung und Teilung auch der natürlichen Ressourcen. Und die universale Gemeinsamkeit der Menschen hebt auch eine strikte Abgeschlossenheit zwischen den einzelnen civitates auf: „Die aber sagen, man müsse sich der eigenen Mitbürger annehmen, nicht aber der Ausländer (externorum), die zerstören die gemeinschaftliche Bindung der Angehörigen des menschlichen Geschlechts zueinander ${ }^{637}$ - man fühlt sich ins 21 . Jahrhundert versetzt.

5. Nicht nur aus Platz-, sondern auch aus Sachgründen folgt nun ein Sprung über Augustinus hinweg zu Thomas von Aquin. Thomas gehört nicht nur, wiewohl Theologe, zu den Klassikern der Rechts- und Staatsphilosophie, er ist auch ein Klassiker des Gemeinwohlbegriffs. Bei ihm finden wir nicht nur aus seinem Werk erschließbare Gemeinwohlvorstellungen, er formuliert und entfaltet selbst den Begriff des Gemeinwohls - bonum commune - als einen Achsenbegriff seiner Rechts- und Staatsphilosophie. Das bonum commune erhält bei Thomas zentrale Bedeutung sowohl für den Gesetzesbegriff, für den Begriff der Gerechtigkeit, für das Handeln der Regierenden und die Grenzen der Gehorsamspflicht der Bürger. Die unruhigen Verhältnisse im Italien des 13. Jahrhunderts, durch vielfache Machtauseinandersetzungen geprägt, mögen dazu beigetragen haben.

a) Thomas definiert das Gesetz - gemeint ist ebenso das menschliche und das natürliche wie das göttliche Gesetz - als ,nichts anderes als eine Anordnung der Vernunft zum gemeinen Wohl, bekanntgemacht von dem, dem die Sorge für die Gemeinschaft obliegt" ${ }^{38}$ Das Gesetz erhält so sein Wesen aus der Hinordnung auf das bonum commune. Und diese Hinordnung bleibt nicht eine bloße Zielausrichtung, sie wird zu einem der Kriterien auch für die Gerechtigkeit des Gesetzes, die ihrerseits eine Bedingung für

${ }^{35}$ Ders., De officiis I, 22. (Übersetzung E.-W. B.)

${ }^{36}$ Ebd., 16, 51. (Übersetzung E.-W. B.)

37 Ders., De officiis III, 6, 28. (Übersetzung E.-W. B.)

38 Thomas v. Aquin, Summa theologiae I-II, qu. 90, art. 4: „nihil aliud quam quaedam rationis ordinatio ad bonum commune, ab eo qui curam communitatis habet, promulgata." (Übersetzung im Text E.-W. B.) 
dessen Verbindlichkeit, seine Verpflichtung im Gewissen ist. ${ }^{39}$ In seiner Gerechtigkeitslehre entwickelt Thomas die bei Aristoteles noch unbestimmt gefaßte allgemeine oder Gesetzesgerechtigkeit zu einer präzis umschriebenen eigenen Gerechtigkeitsart fort, nämlich derjenigen, die das Verhältnis der einzelnen zur politischen Gemeinschaft regelt. ${ }^{40}$ Sie steht einerseits der ausgleichenden Gerechtigkeit gegenüber, die das Beziehungsverhältnis der einzelnen untereinander betrifft, andererseits der austeilenden Gerechtigkeit, die auf das Beziehungsverhältnis der politischen Gemeinschaft zu den einzelnen abstellt. Bezugspunkt und Gegenstand dieser Gerechtigkeitsart ist explizit das Gemeinwohl, dasjenige, was der politischen Gemeinschaft als der Gesamtheit der einzelnen als das Ihrige zukommt und geschuldet ist. Sie erscheint für die Regierenden schöpferisch-gestaltend, architectonice, wie Thomas sagt, für die Bürger vorwiegend ministrative, ausführend, weil den Gesetzen gehorchend. ${ }^{41}$ Schließlich zeigt das bonum commune auch die Grenzen der Gehorsamspflicht gegenüber den Gesetzen auf. Diese Grenze ist nicht schon, wie man vermuten könnte, bei der Ungerechtigkeit des Gesetzes, seinem fehlenden Gemeinwohlbezug, erreicht. Das Gemeinwohl bringt sich hier noch ein weiteres Mal zur Geltung. Bezieht sich die Ungerechtigkeit auf ein menschliches Gut, ist das Gesetz zwar aus sich nicht bindend, aber es kann - um des Gemeinwohls willen - sekundäre Verbindlichkeit erlangen, wenn andernfalls für die Gemeinschaft ein Ärgernis oder grobe Unordnung drohen; denn dann muß der einzelne um des größeren Ganzen willen insoweit auf sein Recht (der Nichtbefolgung) verzichten. Erst wenn das Gesetz gegen ein göttliches Gut, insbesondere gegen göttliche Gesetze verstößt, darf es keinesfalls befolgt werden. ${ }^{42}$

b) Wie aber wird nun dieser Achsenbegriff des bonum commune näher bestimmt? Er bleibt bei Thomas nicht vage und offen, erfährt vielmehr eine deutliche Präzisierung. Dabei tritt zu seiner ethisch-normativen Seite eine wirklichkeitsbezogene, an der conditio humana orientierte Seite hinzu; durch die Verbindung beider wird er offen für eine pragmatische und zu Veränderungen fähige Konkretisierung.

In seiner ethisch-normativen Dimension erscheint das Gemeinwohl, wie schon bei Aristoteles, nicht als überpersönliches, von den einzelnen abgelöstes kollektives Gut; es ist in spezifischer Weise ein Gemeingut, nämlich das gemeinsame Gut aller einzelnen, zu ihrem eigenen, in ihrer Natur angelegten Ziel zu gelangen, dem Glück des vollendeten Lebens. Und dies kann nur in gemeinschaftlichen Lebenszusammenhängen erreicht werden. Das ist zwar durchaus Aristoteles, aber nicht mehr reiner Aristoteles, denn das letzte Ziel, auf das die menschliche Natur angelegt ist, ist für den christlichen Theologen Thomas theologisch bestimmt: die ewige Glückseligkeit in der assimilatio Dei. ${ }^{43}$ Doch wird das Erreichen dieses Endziels im irdischen Leben nur vorbereitet, nicht schon herbeigeführt, und das irdische Gemeinwohl befaßt sich mit den natürlichen Be-

39 Ebd., qu. 96, art. 4.

$40 \mathrm{Vgl}$. ebd., II-II, qu. 58, art. $6 \mathrm{f}$.

4I Vgl. ebd., II-II, qu. 58, art. 6 resp.

42 Vgl. ebd., I-III, qu. 96, art. 4 resp.

43 Ebd., qu. 3, art. 8 resp.: „Ultima et perfecta beatitudo non potest esse nisi in visione divinae essentiae." 
dingungen dafür. Die irdisch-natürliche Aufgabe wird so zwar theologisch fundiert, aber nicht theologisch okkupiert, sie bleibt als natürliche erhalten.

Die der gegebenen Wirklichkeit zugewandte Seite des Gemeinwohls betrifft die Berücksichtigung geschichtlich-politischer Gegebenheiten und des Umstandes, daß die Gesetze den Menschen gegeben werden, nicht wie sie sein sollen, sondern wie sie sind. Sie müssen deshalb der conditio humana Rechnung tragen und für die empirischen Menschen erfüllbar sein. Thomas stellt und verneint die Frage, ob das menschliche Gesetz alle Laster, omnia vitia, verbieten müsse. ${ }^{44}$ Ungeachtet der von ihm festgehaltenen Ausrichtung des Gemeinwohls auf das vollendete tugendhafte Leben billigt er es, wenn die Gesetze nur die schwereren Vergehen untersagen, die anderen schaden und ohne deren Verbot eine menschliche Gesellschaft keinen Bestand haben könnte. ${ }^{45}$ Das ist ein richtungweisender Ansatz für die Erkenntnis der Eigenständigkeit des positiven Rechts gegenüber Ethik und Moral. Doch wird damit die ethisch-normative Dimension des bonum commune nicht aufgegeben? Thomas tut dies nicht, und hier kommt das dynamischbewegliche Moment des Gemeinwohlbegriffs ins Spiel: „Das menschliche Gesetz", sagt er, „hat zwar die Intention, die Menschen zur Tugend hinzuführen, aber nicht von einem Augenblick zum andern, sondern Schritt für Schritt. Deshalb legt es der Menge der Unvollkommenen nicht sofort auf, was für die bereits Tugendhaften gilt“. Der Grund dafür: „Andernfalls nämlich würden sich die Unvollkommenen in ihrem Unvermögen, Gebote dieser Art zu befolgen, noch zu schlimmeren Übeln hinreißen lassen. “"

c) Thomas präzisiert nun den Gemeinwohlbegriff nicht nur in dieser Weise, er liefert darüber hinaus auch ein ontologisch-philosophisches Fundament für ihn. Zum einen bestimmt er den Seinsstatus menschlicher Gemeinschaften, insbesondere auch der politischen Gemeinschaft - modernen sozialwissenschaftlichen Erkenntnissen, etwa Hermann Hellers, vorausgreifend -, als den einer unitas ordinis, einer (nur) Wirk- und Ordnungseinheit, nicht einer irgendwie substantiellen Einheit. ${ }^{47}$ Nur die einzelnen Menschen sind für sich seiend (per se subsistens), die Gemeinschaft besteht lediglich als notwendige Beziehungsordnung, eine Vielheit von Menschen unter einer Ordnung zusammengefaßt. Daraus folgt dann aber, daß das Ziel einer solchen Gemeinschaft nicht etwas von dem Gut und Ziel der einzelnen Abgelöstes sein kann, sondern nur das gemeinsame Ziel der Glieder dieser Gemeinschaft umfaßt.

Zum andern begründet er - entsprechend der Metapher von Ganzem und Teil - den Vorrang und die Höherwertigkeit des Gemeinwohls gegenüber dem Einzelwohl. Darin liegt kein Widerspruch. Wenn der einzelne als ein im vollen Sinn auf Gemeinschaft hin geordnetes Wesen das, was er selbst ist und sein kann, nicht auf sich gestellt, sondern nur als Glied und Teil von Gemeinschaften erreichen kann, hängt in der Tat sein Sein-Können und sein individuelles Wohl vom Bestand und der guten Ordnung der Gemeinschaft ab. Rechte und Belange der einzelnen können dann nur im Rahmen einer Gemeinschaftsordnung und auf deren Grundlage begründet und gesichert werden. Nur unter dieser Voraussetzung läßt sich übrigens auch der Einsatz und gegebenenfalls das Opfer von Gesundheit

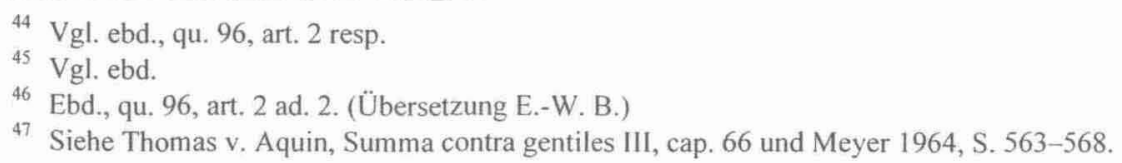


und Leben der einzelnen für die Gemeinschaft rechtfertigen. Daß daraus kein Umschlag in ein Kollektiv entsteht, bei dem die einzelnen zum bloßen Zubehör des Ganzen werden, liegt in der Gleichheit des Ziels der politischen Gemeinschaft und der einzelnen, von dem Thomas ausgeht. ${ }^{48}$ Hinzu kommt, daß Vorrang wie Höherwertigkeit des Gemeinwohls nach Thomas jeweils nur auf der gleichen Ebene bestehen; die Hinordnung auf Gott transzendiert die politische Gemeinschaft und Gemeinschaftsbindung, insoweit ist der einzelne unmittelbar zu Gott und nur Glied der Kirche. ${ }^{49}$

6. Bevor wir wieder einen Sprung - zu Thomas Hobbes hin - machen, sei noch ein Blick auf die spanische Spätscholastik geworfen. Sie bewegt sich weithin auf den von Thomas vorgezeigten Bahnen; dessen Autorität war nicht nur für Dominikaner, wie Franz von Vitoria, sondern auch für Jesuiten, wie Francisco Suárez, unbestritten. ${ }^{\text {So }}$ Wie Thomas argumentiert man im Rahmen und von den Voraussetzungen des christlichen Weltbildes her. Aber es zeigt sich doch - mitveranlaßt durch die neuen Gegebenheiten, wie die spanische Herrschaft in Amerika und die Spaltung der Glaubenseinheit in der Reformation - eine deutliche Akzentverschiebung in der Ausrichtung des Gemeinwohlbegriffs: die stärkere Orientierung auf Frieden und Sicherheit hin und die Einbringung und Aktualisierung eines bereits menschenrechtlich konzipierten Freiheitsgedankens.

Eher zurückhaltend ist, was den Gemeinwohlbegriff angeht, noch Franz von Vitoria. Das Um-willen der politischen Gemeinschaft sind für ihn die natürlichen Lebenszwecke der Menschen, die diese Gemeinschaft bilden, ihre felicitas politica. Dabei nennt er öfters und mit einem gewissen Vorrang ,pax et securitas“. ${ }^{\text {. }}$ " Aber er bezieht diese Zwecke zugleich in das hauptsächliche und umgreifende Ziel der politischen Gemeinschaft ein, die Erreichung des tugendhaften Lebens. Eben darauf bleibt das bonum commune bezogen, es ist nicht nur mit dem bonum naturale, sondern auch dem bonum morale der Bürger verbunden. Pax und securitas haben so Bedeutung als Medium daraufhin, dienen auch einem weiteren, über sie selbst hinausweisenden Ziel.

Deutlicher in dieser Hinsicht wird Francisco Suárez. Das irdische Gemeinwohl, die felicitas politica, bestimmt er nicht mehr voll im Sinne des vollendeten tugendhaften Lebens. Äußerer Friede und Gerechtigkeit im Sinne der Rechtspflege werden - Suárez hat die religiös-politischen Auseinandersetzungen des 16. Jahrhunderts in Europa vor Augen - das erste wesentliche Moment des Gemeinwohls; die Beobachtung der guten Sitten wird demgegenüber akzessorisch, sie gehört nur insoweit dazu, als sie für das äußerliche Zusammenleben der Menschen und die Erhaltung der (körperlichen) Natur

48 Thomas v. Aquin, De regno II, 3, S. 45-46: „Idem autem oportet esse iudicium de fine totius multitudinis, et unius."

49 Ders., Summa theologiae I-II, qu. 152, art. 4 ad, 3, auch ebd. I-II, qu. 21, art. 4 ad. 4.

50 Vitoria wirkte während seiner Zeit an der Universität Paris (1508-1522) daran mit, daß für den theologisch-philosophischen Unterricht anstelle der Sentenzen des Petrus Lombardus, die über Jahrhunderte als maßgebendes Lehrbuch fungierten, nunmehr die Summa theologiae zugrunde gelegt wurde. Er selbst nahm sich Thomas v. Aquin zum Vorbild, ohne ihn unkritisch zu reproduzieren, und ein beträchtlicher Teil seines Werkes besteht in Kommentaren zur Summa theologiae. Für Suárez gibt Thomas v. Aquin, in seinem grundlegenden Werk De legibus als „divus Thomas" tituliert, den maßgeblichen Orientierungspunkt ab.

51 Nachweise dazu bei Deckers 1991, S. 300 f. 
erforderlich ist. Ziel der politischen Herrschaftsgewalt ist, sagt Suárez, daß die einzelnen Menschen ,,in Frieden und Gerechtigkeit leben und mit den Gütern, die zur Erhaltung und Annehmlichkeit des körperlichen Lebens dienen, hinreichend ausgestattet sind, und ebenso auch mit derjenigen Integrität der Sitten, die für diesen äußeren Frieden, die Wohlfahrt des Staates und die Erhaltung der menschlichen Natur notwendig ist ${ }^{\text {" }}$. 22 Die politische Aufgabe des Staates tritt in den Vordergrund, die ethische ein Stück weit zurück. An der Aufgabe der Gesetze, wie Suárez sie sieht, wird dies weiter verdeutlicht. Diese streben, dem Ziel des Staates gemäß, ,nicht die volle Integrität des sittlichen Verhaltens an, die den Menschen zum guten Menschen macht, sondern nur eine gewisse äußere Befolgung von Regeln, die ihn zu einem guten Bürger machen“. 53

Der Freiheitsgedanke, ja das Freiheitsprinzip als Teil des Gemeinwohls, tritt am stärksten bei Las Casas, dem Kämpfer für die Rechte der Indios, hervor. Freiheit und Freiheitsfähigkeit sind für ihn ein Grundprinzip, das in der Schöpfung Gottes enthalten ist. Beim Ursprung des vernunftbegabten Geschöpfs, heißt es, wurden alle frei geboren und Gott hat allen gleichermaßen das freie Entscheidungsvermögen zugestanden. ${ }^{54}$ Diese ursprüngliche Freiheit der Menschen führt zum Recht der Selbstherrschaft jedes Volkes kraft Naturrechts. Die Freiheit der einzelnen impliziert die Freiheit der Völker und damit deren eigenes Recht zur Begründung und Konstituierung politischer Herrschaft und deren Organisation. Dieses Recht ist ein notwendiger Bestandteil des gemeinen Wohls. Die Konsequenzen gehen noch weiter. Weil es sich bei jedem Volk vom Ursprung her um ein freies Volk handelt, ,geschieht die Regierung einer Gemeinschaft freier Menschen wegen der freien Menschen, also muß sie auf das Wohl und den Nutzen dieser hin geordnet werden" ${ }^{55}$ So ist die Wahl der Könige und derer, die freie Menschen und Völker regieren sollen, Sache just derer, die regiert werden sollen, und dies kraft natürlichen und kraft Völkerrechts. ${ }^{56}$ Hier kommt erneut die institutionelle und organisatorische Seite des Gemeinwohls zum Vorschein, die wir bei Aristoteles und Cicero fanden, aber nun in anderer, auf Freiheit und Selbstbestimmung der einzelnen und des Volkes bezogener Weise. Und Las Casas geht noch einen Schritt weiter. Die Herrschaft der Könige und Fürsten stellt nur eine Amtsgewalt dar, sie sind ,im eigentlichen Sinn gesprochen, nicht Herren ihrer Königreiche [...] sondern nur Regenten, Vorsteher und Verwalter des Gemeinwesens ${ }^{4} .{ }^{57}$ Der Fürst hat über die Untertanen ,nicht eine eigene Gewalt, vielmehr nur die Gewalt des Gesetzes, das am Allgemeinwohl zu orientieren ist; er herrscht nicht im eigentlichen Sinn, sondern verwaltet das Volk durch die Gesetze [...] so bleiben die Bürger frei, da sie nicht dem Menschen, sondern dem Gesetz gehorchen". ${ }^{8}$ Dies liest man im 16. Jahrhundert in Spanien!

52 Suárez 1975, III, c. 11 n 7. (Übersetzung E.-W. B.)

${ }^{53}$ Suárez 1972, I, c 13 n 2: , ad quem finem etiam ordinantur civiles leges, non ergo intendunt veram probitatem morum quae fecit hominem bonum, sed solum quandam exteriorem observantiam, quae fecit bonum civem." (Übersetzung im Text E.-W. B.)

${ }^{54} \mathrm{Vgl}$. Las Casas 1992, S. 562 und Las Casas 1996, S. 47.

55 Ders. 1996, S. 52; vgl. Las Casas 1992, S. 570.

${ }_{56}$ Vgl. ders. 1996, S. 226 und Las Casas 1992, S. 447.

57 Ders. 1997, S. 207; siehe auch Las Casas 1990, S. 68.

${ }^{58}$ Ders. 1997, S. 213; siehe auch Las Casas 1990, S. 84. 
7. Bei Thomas Hobbes begegnet uns nun ein Paradigmenwechsel in der Bestimmung und Fundierung von Gemeinwohl. Zeithistorisch veranlaßt war er durch die Situation der europaweit ausgetragenen konfessionellen Bürgerkriege und die fortdauernden Machtauseinandersetzungen zwischen Krone und Parlament in England, die in der Herrschaft Cromwells (1649-1658) einen Höhepunkt erreichte. Hobbes argumentiert nicht mehr von einer vorgegebenen natürlichen Ordnung her, die als Schöpfungsordnung in Gott ihren Urheber hat, vielmehr sucht er möglichst voraussetzungslos die Ordnung des Zusammenlebens der Menschen in strengen Vernunftschlüssen - more geometrico - zu begründen und die notwendigen, nicht hintergehbaren strukturellen Merkmale dieser Ordnung aufzuweisen. ${ }^{59}$ Hobbes nimmt seinen Ausgangspunkt einerseits beim einzelnen, auf sich gestellten ungebundenen Individuum in seiner existentiellen Bedürftigkeit, es ist das letzte, nicht mehr teilbare Element des politischen Körpers; anderseits bei der empirischen, in der Wirklichkeit vorfindlichen Natur der Menschen, d. h. deren tatsächlichen Verhaltensweisen und Motivationen. Beides bildet die Konstruktionsbasis, das Material, anhand dessen zweckrationale Vernunftschlüsse (dictamina rectae rationis) Bedingungen und Eigenart der politischen Ordnung stringent aufweisen.

Diese Vernunftschlüsse führen zu dem bekannten Staatsgründungsvertrag jedes mit jedem, indem jeder einzelne sein natürliches Recht auf alles, was seinem Leben und seiner Erhaltung dient, insoweit einer einheitlichen Entscheidungsinstanz - sei es Monarch oder Parlament - einräumt, als dies für die Erreichung von Frieden und Sicherheit notwendig erscheint, und demgemäß deren Entscheidungen als seine eigenen anerkennt. ${ }^{60}$ Das ist der Ausgangspunkt. Was nun aber zur Erreichung von Frieden und Sicherheit notwendig ist, ist nicht weniger als eine - Hobbes leitet dies Stück für Stück folgerichtig her ${ }^{61}$ - umfassende, höchste und souveräne Entscheidungsgewalt, die ihrerseits einer rechtlichen Bindung nicht mehr unterliegt - ein sumum imperium und imperium absolutum, wie Hobbes selbst sagt. ${ }^{62}$ Ist damit aber das Um-willen der Staatsgründung, die darin enthaltene Gemeinwohlvorstellung von Frieden und Sicherheit für die im Staat zusammenlebenden Menschen nicht aufgezehrt, untergegangen zugunsten einer absoluten, inappelablen Staatsgewalt, die nach Belieben handeln kann?

59 Als methodisches Paradigma fungiert - Fascinosum der Zeit - das Uhrwerk. Wie dieses in seiner Funktionsweise, Zusammensetzung und Gesetzlichkeit nur erkannt werden kann, wenn man es in seine letzten Teile zerlegt und von dort her wieder aufkonstruiert, so will Hobbes entsprechend beim politischen Körper vorgehen, vgl. Hobbes 1983, S. 77-84 (praefatio ad lectores).

${ }^{60}$ Hobbes 1983, S. 133: (V, 6): Ziel des Vertrages ist, ,ut circa ea quae ad pacem et defensionem sunt necessaria, una omnium sit voluntas". Deshalb muß jeder einzelne seinen Willen dem eines anderen so unterwerfen, ,ut pro voluntate omnium et singulorum, habendum sit, quicquid de iis rebus quae necessariae sunt ad pacem communem ille voluerit". Im Leviathan tritt der Gedanke, der Autorisierung und Repräsentation hinzu: der Vertrag ist „as much as to say to appoint one Man, or Assembly of men, to beare their Person [...] and acknowledge himselfe to be the Author of whatsoever he that so beareth their Person, shall Act, or cause to be Acted, in those things, which concern the Common Peace and Safetie [...]" (Hobbes 1991, S. 120 [c. 17]).

61 Vgl. Hobbes 1983, S. 135-145 (VI, 1-15) und ders. 1991, c. 18.

${ }^{62}$ Vgl. ders. 1983, S. 141-144 (VI, 13): ,in omni civitate perfecta [...] esse summum in aliquo imperium, quo maius ab hominibus iure conferri non potest. [...]. Imperium autem quo maius ab hominibus in hominem transferri non potest, vocamus absolutum." (S. 141 f.) 
Es gibt nicht wenige, die Hobbes gerne so lesen würden, aber man greift damit zu kurz. Nicht nur daß Hobbes die Beziehung von Schutz und Gehorsam - protection and obedience - als Grundbedingung für Staatsentstehung und Staatsbestand immer festhält, er spricht auch ausdrücklich und einläßlich von den Pflichten derer, die das sumum imperium ausüben und verwalten (de officiis eorum qui summum imperium administrant). ${ }^{63}$ An dieser Stelle entwickelt er Gemeinwohlvorstellungen im Hinblick darauf, wie denn der Zustand des äußeren Friedens näher auszugestalten sei. Dabei verwendet und definiert er auch den Begriff der salus publica. Allerdings handelt es sich bei diesen Pflichten um Verpflichtungen nach natural law, gemäß der rechten Vernunft. ${ }^{64}$ Das sind für Hobbes moralische Verpflichtungen, vor allem Gott gegenüber; sie sind rechtlich nicht einforderbar.

Hieran wird Hobbes' Grundkonzept noch einmal deutlich: Rechtlich gesehen bedarf es, um die Menschen voreinander gegenüber ihrer (potentiellen) Gewalttätigkeit zu schützen, einer einheitlichen, allzuständigen und inappelablen Entscheidungsgewalt; dies ist die minimum condition für Frieden und Sicherheit. Auf dieser Grundlage aber behalten ethisch-moralische Pflichten ihre Relevanz, ja sind notwendig, um den erstrebten Zustand zu erreichen. Nur dürfen sie nicht als rechtliche, und das heißt rechtlich einklagbare Pflichten (vor wem?) qualifiziert werden, weil dann die höchste und einheitliche Entscheidungsgewalt ihre Souveränität verliert und sich (wieder) auflöst. Bei Hobbes stehen die lex naturalis und die leges naturales nebeneinander. Die erste enthält die dictamina rectae rationis für die Erlangung von Frieden und Sicherheit, ${ }^{65}$ die letzteren enthalten die Regeln und Grundsätze, die von den Einzelnen wie den Herrschern zu beobachten sind, damit der äußere Frieden auch zu einem inhaltlichen Frieden wird. ${ }^{66}$ Hobbes unterscheidet und trennt also ausdrücklich rechtliche und ethische Gebote, die vorher kaum geschieden waren. Die Gemeinwohlorientierung und -bindung der Regierenden verläßt den rechtlichen und wechselt in den ethisch-moralischen Bereich, behält aber dort ihre verpflichtende Kraft, zumal in einem Christian Commonwealth, für das nach Hobbes - die Prämisse that Jesus is the Christ und damit die Verantwortung Gott gegenüber gültig ist.

Wie aber sieht nun die so verortete Gemeinwohlbindung inhaltlich aus? Für Hobbes gilt als oberste Maxime: salus populi suprema lex. Salus populi umfaßt dabei nicht nur eine irgendwie geartete Erhaltung des Lebens, sondern soweit möglich ein glückliches Leben (vita beata). Darunter versteht Hobbes aber nicht mehr ein vollendetes tugendhaftes Leben, sondern ein so angenehmes diesseitiges Leben, wie es die menschliche Natur gestattet. Er konkretisiert dies, indem er die Frage stellt: salus publica in quo consistit? Seine Antwort ist von klassischer Prägnanz:

${ }^{63}$ Vgl. ebd., S. 194 (XIII, Überschrift).

${ }^{64}$ Vgl. ebd., S. 195 (XIII, 2): „[...] officii tamen eorum est rectae rationis, quae lex est naturalis, moralis et divina, quantum possunt in omnibus obedire."

${ }^{65}$ Vgl. ebd., S. 99 (II, 1): „Est igitur lex naturalis [...] dictamen rectae rationis circa ea, quae agenda vel omittenda sunt ad vitae membrorumque conservationem, quantum fieri potest, diuturnam."

${ }^{66}$ Vgl. ebd., S. 107-121 (III), 194-197 (XIII, 1-6); Hobbes 1991, c. 14, 15; sowie Schilling 1966, S. 288-292. 
„Commoda civium quae hanc tantum vitam spectant, in quatuor genera distribui possunt: 1. ut ab hostibus externis defendantur; 2. ut pax interna conservetur; 3 . ut quantum cum securitate publica consistere potest, locupletentur; 4 . ut libertate innoxia perfruantur. “67

Hobbes begründet dies wie folgt: Mehr nämlich, als daß die Bürger vor äußeren und Bürgerkriegen sicher sind und ihr durch ihren Fleiß erworbenes Vermögen ruhig genießen können, können die (souveränen) Herrscher für dies bürgerliche Glück (felicitatem civilem) nicht tun. ${ }^{68}$ Die nähere Erläuterung, die er dazu gibt, ${ }^{69}$ zeigt, wie die Ausrichtung auf den formellen Rechtsstaat und staatliche Wohlfahrtsförderung diesen Gemeinwohlbegriff prägt und auch begrenzt; die moralische Vervollkommnung der Menschen gehört nicht dazu, sie betrifft deren himmlisches Heil. Auffällig ist allerdings, daß diese durchaus säkulare, auf die Bedürfnisnatur der Menschen abstellende Fassung des Gemeinwohlbegriffs keine Maxime für die Ordnung der Güterzuteilung an die einzelnen umfaßt. Hobbes verhält sich dazu nicht und hinterläßt insoweit ein offenes, sehr verschiedener Ausfüllung zugängliches Feld.

8. Dies wird ersichtlich anders bei einem Antipoden von Hobbes, der freilich zu einem Teil auf seinen Schultern steht, John Locke. Auch Locke geht zur Staatsbegründung von einem staatlosen Naturzustand aus, indem die einzelnen sich als gleiche und freie Individuen ohne übergreifende Ordnungsgewalt einander gegenüberstehen und jeder für sich die Bedürfnisse des Lebens, die commoda vitae, zu befriedigen sucht. Ein Element dieses Naturzustandes ist die Unsicherheit. Aber sie entwickelt sich, anders als bei Hobbes, nicht zur allgemeinen Existenzfurcht und einem Zustand des bellum omnium contra omnes. Der Naturzustand ist bei Locke bereits strukturiert, enthält eine - wenngleich ungesicherte - Ordnung durch die natural laws, die sich aus der natürlichen Vernunft (natural reason) ergeben. ${ }^{70}$ Diese ist freilich, hier zeigt sich die Umdeutung der Begriffe, ohne metaphysischen Bezug. Sie stellt sich als die gemeinsame, im common sense evidente Ansicht über das zum angenehmen Leben Notwendige und Nützliche dar - eine individualistisch-eudämonistisch orientierte „reasonableness“. Das Um-willen des Staates, sein common good, ist daher nicht abstrakt Frieden und Sicherheit zu erreichen, um überhaupt zu einer Ordnung zu kommen, wie bei Hobbes, sondern den durch die natural laws bereits geformten Naturzustand $z u$ einem sicheren zu machen, seine Gefährdungen zu beseitigen. ${ }^{71}$ Der Inhalt des public good, das, was die politische Gemeinschaft zu sichern und zu gewährleisten hat, ist die durch natural laws bereits geschaffene Ordnung, sie liegt dem Staat voraus und bestimmt sein Gemeinwohl.

Wie sieht diese Ordnung des Naturzustands aus? Sie wird bestimmt durch die Sicherung von Freiheit und Eigentum. Auf der Grundlage der Freiheit und Gleichheit der einzelnen ergeben sich zunächst elementare Unterlassungs- bzw. Nichtstörungspflichten als Pendant der den einzelnen zukommenden natürlichen Rechte: Die Nichtbeeinträch-

${ }^{67}$ Ders. 1983, S. 197 (XIII, 6).

${ }^{68}$ Ebd.

69 Vgl. ebd., S. 197-204 (XIII, 7-17).

${ }^{70}$ Diese natural laws werden in den Two Treatises of Government II, §§ 4-14, 24-52 im einzelnen hergeleitet und diskutiert.

${ }^{71}$ Siehe Locke 1988, §§ 123-126, 131 . 
tigung von Leben, Gesundheit, Freiheit der Person und des Handelns, insonderheit der Verfügung über das Eigentum. ${ }^{72}$ Hinzu treten Vertragsschluß und Vertragsbindung als Elemente der Beziehungsordnung unter den Menschen. Entscheidend für die positive Struktur der Naturzustandsordnung werden dann Eigentumsrecht und Eigentumserwerb, in ihnen wird die soziale Leitidee des Naturzustands und der Naturzustandsgesellschaft greifbar. Das Recht auf Eigentum folgt aus dem Recht der Selbsterhaltung, es umfaßt das Recht zur Aneignung von Gütern zwecks Sicherung des eigenen Lebensunterhalts. Zugleich erfährt dieses Recht eine Begrenzung durch den biblisch abgeleiteten Widmungszweck der Erdengüter an die Menschen insgesamt: eine Aneignung von Gütern, die zu deren Verderbnis führt, weil sie vom Aneignenden selbst nicht verbraucht werden können, ist gegen das natürliche Recht. Insoweit werden Autarkievorstellungen fortgetragen. $^{73}$

Eigentum und Eigentumsordnung werden freilich nicht mehr primär statisch gesehen, als institutionelle Zuteilungs- und Anteilsordnung - denken wir an Aristoteles -, sondern vom Erwerb her. Locke hat die gerade in England sich ausbildende, auch in die Kolonialgebiete ausgreifende Erwerbs- und Handelsgesellschaft vor Augen. ${ }^{74}$ So gelangt er - die wirtschaftlichen Vorgänge und Veränderungen der Zeit reflektierend - zu einem neuen und weittragenden Ansatz. Grund und Titel des Eigentums wird der Aneignungsvorgang, Eigentum erscheint als Ertrag und Ausdruck von Arbeit. Locke verbindet so Arbeit mit Eigentum und Eigentum mit Person und Freiheit, und zugleich bereitet er der dynamischen Ausdehnung von Eigentum das Feld: ${ }^{75}$ die Anhäufung von Eigentum durch Arbeit begründet Reichtum. Und da Arbeit und Arbeitskraft höchstes Eigentum der Person sind, kann über sie verfügt, kann die Arbeitskraft veräußert werden. Mit solchem Verkauf der Arbeit geht dann - dies liegt in der Konsequenz - auch das Werk der Arbeit in das Eigentum dessen über, der die Arbeit des anderen zu Eigentum erworben hat. ${ }^{76}$ Dies bedeutet die Legitimation einer Eigentümergesellschaft, die den arbeitsteiligen industriewirtschaftlichen Prozeß und die Abhängigkeit der Arbeit zu ihrer Grundlage hat. Locke vollendet diese Legitimation noch, indem er den durch das natural law gedeckten Eigentumserwerb von der Beschränkung auf die Sicherung der eigenen Lebensbedürfnisse befreit. Die Begründung ist ein (dialektisches) Meisterstück, mit dem die alte Ordnung formell unberührt bleibt, aber materiell aus den Angeln gehoben wird. Zwar gilt die ursprüngliche Begrenzung des Eigentumserwerbs fort, sagt Locke, aber: Gold und Silber dürfen angehäuft werden, denn sie verderben nicht. ${ }^{77}$ Über

${ }^{72}$ Vgl. ebd., $\$ \S 4-6$.

73 Vgl. ebd., $\$ \$ 24-25,31$.

74 Siehe dazu Trevelyan 1948, S. 206-226, 265-284.

75 Vgl. ebd., $\$ \$ 26-27$.

${ }^{76}$ Dies wird von Locke teils angedeutet, vgl. § 28, teils auch in seiner Argumentation vorausgesetzt, vgl. dazu Macpherson 1973, S. 243 ff.

77 Vgl. ebd., $\S \S 45-46$. Die Kernsătze lauten: „Again, if he would give his Nuts for a piece of Metal, pleased with its colours; or exchange his Sheeps for Shells, or Wool for sparkling Pebble or a Diamond, and keep those by him all his Life, he invaded not the Rights of others, he might heap up as much of these durable things as he pleased; the exceeding of the bounds of his just Property not ly- 
Geldverkehr und Geldwirtschaft wird die Güterakkumulation in der Form der Kapitalakkumulation freigesetzt, mit allem, was daraus folgt. Locke rechnet dazu auch die implizite Übereinkunft über Besitzungleichheit und die sich darauf gründenden Unterschiede in der Gesellschaft. ${ }^{78}$

Dies alles gehört - deshalb war dieser Exkurs notwendig - zur Ordnung des Naturzustandes von Freiheit und Eigentum. Deren Erhaltung und Sicherung ist die Aufgabe der staatlichen Ordnung, begründet und begrenzt sie. Locke faßt sie unter dem Kürzel ,preservation of property" zusammen. ${ }^{79}$ Preservation of property erscheint als das common good der political society. ${ }^{80}$ Es wird durch den Staat und seine Gesetze nicht eingeführt, sondern liegt ihnen voraus; die Gesetze - promulgated, standing laws - haben dies nur im einzelnen auszuformen und zu verdeutlichen. Die gute Ordnung, das Gemeinwohl des Staates besteht somit darin, Schutzorganisation einer selbstnützigen Eigentümererwerbsgesellschaft zu sein. Ein Zugriff auf die Güterzuordnung und Güterverteilung hat er nicht. Es gibt keine Gründe des Gemeinwohls, die ihm einen solchen Zugriff verschaffen können, weil das Gemeinwohl ja eben darin besteht, die naturrechtlich legitimierte Eigentümererwerbsgesellschaft und ihre Ordnung zu erhalten, auch wenn das eine Klassenherrschaft aus sich heraussetzt. Nicht umsonst wurde gerade Locke später zu einem Anknüpfungspunkt für Karl Marx.

9. Von der besitzindividualistischen Gemeinwohlvorstellung John Lockes nun wiederum ein Sprung zu Immanuel Kant, dem Philosophen der Freiheit. Er zieht die von Hobbes begonnene Linie in einer Richtung weiter aus, stellt sie zugleich auf eine neue Grundlage und führt sie von daher zu einem Abschluß.

Die Notwendigkeit einer staatlichen Ordnung, die eine höchste Bestimmungs- und äußere Zwangsgewalt in sich vereinigt, ergibt sich für Kant aus einem zweifachen Begründungsansatz. Zum einen folgt sie aus der naturalen, erfahrungsbestätigten Befindlichkeit der Menschen, ihrer ungeselligen Geselligkeit und der Zwiespältigkeit in ihrem Verhalten zwischen Vernunftwesen und Tier. Hier wandelt Kant auf den Spuren von Hobbes. Die für ungebundene Freiheit so eingenommenen Menschen zwingt die Not, und zwar die, welche sich die Menschen untereinander selbst zufügen (können), in die bürgerliche Vereinigung, den Staat, einzutreten; sie müssen ein Gehege der Freiheit, die gesetzliche Freiheit, suchen, weil sie in wilder Freiheit nicht lange nebeneinander bestehen können. ${ }^{81}$ Zum andern und zugleich erscheint der Staat, abstrakt betrachtet, a priori notwendig als die Bedingung für die Geltung des Rechtsgesetzes, das Freiheit als äußere Freiheit erst schafft und dadurch das Leben in sittlicher Freiheit - nach dem, was mir recht und gut dünkt, ohne von der Meinung anderer abzuhängen - ermöglicht. ${ }^{82}$ Dies setzt eben den öffentlich-gesetzlichen Zustand voraus, der jedem das Seinige zuerkennt und durch den Richter durchsetzt.

\footnotetext{
ing in the largeness of his Possession, but the perishing of any thing uselesly in it." ( $§ 46$, S. 300; Hervorhebg. i Orig.).

78 Vgl. ebd., $§ 50$.

79 Ebd., § 123.

80 Vgl. ebd., § 135.

81 Vgl. Kant 1977a, S. 40.

82 Vgl. ders. 1977b, Teil 2, §44, S. 431 f.
} 
So ist es nicht das Telos der menschlichen Natur, eine Sozialität oder Bedürftigkeit, die den Staat begründet und legitimiert, sondern das Freiheitsprinzip, die Freiheit im und aus dem gesetzlichen Zustand anstelle wilder gesetzloser Freiheit. Eben deshalb definiert Kant den Staat als „Vereinigung einer Menge von Menschen unter Rechtsgesetzen“ ${ }^{83}$ d. h. Gesetzen, die, dem kantischen Rechtsbegriff gemäß, die Bedingungen der Freiheit für alle und einen jeden beinhalten.

Von dieser Begründung und Legitimation her bestimmt sich der Charakter des Staates und das, was das gemeine Wohl, die salus publica, ausmacht. Für Kant fließen hier die organisatorische und die inhaltliche Seite ineinander. Organisatorisch geht es um die Elemente einer Staatsverfassung a priori, d. h. einer Verfassung nach Rechts- $(=$ Freiheits)gesetzen. Zu ihr gehören drei Prinzipien:

(1) die Freiheit eines jeden als Mensch, d. h. die Anerkennung der Subjektstellung, des Rechtes der Menschheit im einzelnen Menschen; das führt auch zur Begrenzung der Staatszwecke zugunsten der Selbstbestimmung des einzelnen, gerichtet gegen das Prinzip wohlfahrtsstaatlicher Landesväterlichkeit. ${ }^{84}$

(2) die Gleichheit jedes einzelnen als Untertan, die sich in der Rechtsgleichheit und der gleichen Unterworfenheit eines jeden unter die öffentliche Gewalt zeigt. Hier ist der Hieb gegen Ständeordnung, gegen Privilegien und jede andere als staatliche Herrschaft und Gesetzgebungsmacht unübersehbar. ${ }^{85}$

(3) die Selbständigkeit der Bürger als aktive Beteiligung an den Staatsangelegenheiten, insbesondere der Gesetzgebung. Sie kommt all denen zu, die ihr eigener Herr (sui iuris) sind, d. h. ihre Existenz und Nahrung aus Selbständigkeit, nach eigenem Betriebe, wie Kant sagt, erhalten, nicht nach der Verfügung anderer ${ }^{86}$ Der Grund für diese Begrenzung, deren Zeitabhängigkeit unübersehbar ist, liegt nach meiner Einschätzung allerdings weniger in einem Besitzdenken als in Kants Freiheitsdenken: Unabhängigkeit, der Selbststand im bürgerlichen Leben, ist die Bedingung der , freien' Bestimmung über die Freiheitsordnung.

Inhaltlich bringt sich die im Organisatorischen schon angelegte Begrenzung der Staatszwecke und Staatsaufgaben noch einmal zur Geltung. Der Zweck a priori des Staates ist allein die Verwirklichung und Sicherung des Rechtsgesetzes, die Verfolgung empirischer Zwecke, gerichtet auf die Glückseligkeit der Bürger, gehört nicht dazu. Kant nimmt dies auch ausdrücklich in seine Definition der salus publica hinein: Das öffentliche Heil, sagt er, ,ist gerade diejenige gesetzliche Verfassung, die jedem seine Freiheit durch Gesetze sichert: wobei es ihm unbenommen bleibt, seine Glückseligkeit auf jedem Wege, welcher ihm der beste dünkt, zu suchen, wenn er nur nicht jener allgemeinen gesetzmäßigen Freiheit, mithin dem Rechte anderer Mituntertanen, Abbruch tut ${ }^{4}{ }^{87}$ Fraglich ist, inwieweit damit die Wohlfahrtsfunktion als Bestandteil des Gemeinwohls überhaupt, oder nur als Funktion a priori ausgeschlossen wird, indes abhängig von Um-

${ }^{83}$ Ebd., S.431.

84 Vgl. ders. 1977c, S. 145 f.

${ }^{85}$ Vgl. ebd., S. $146 \mathrm{f}$.

${ }^{86}$ Vgl. ebd., S. 150 ff. und Kant 1977b, Teil 2, § 46, S. 432 ff.

${ }^{87}$ Ders. 1977c, S. 155. 
ständen und Konstellationen durchaus zulässig sein kann. Diese Frage zu entscheiden, ist schwierig. Kant hat die Ausübung der gesetzgebenden Gewalt über das Postulat hinaus, das im gesetzgebenden Willen ein jeder über alle und alle über einen jeden beschließen müssen, nicht weiter ausgeführt. Einige Bemerkungen sprechen indes dafür, $\mathrm{da} ß$ die Wohlfahrtsfunktion als subsidiäre, um generell eine Wohlhabenheit der im Staat vereinigten Menschen zu erhalten und den Staat als das gemeine Wesen - auch gegenüber auswärtiger Bedrohung - zu sichern, offengehalten werden sollte. ${ }^{88}$ Wie dem auch sei, jedenfalls fehlt in Kants Gemeinwohlvorstellung eine über die Freiheit hinausgehende positive Sozialidee. Das Woraufhin der Freiheit, die Stelle der alten eudaimonia, bleibt leer. Kant verlegt dies in die Freiheit selbst, verstanden als die Autonomie der einzelnen, womit der überindividuelle Bezug entfällt. Das hat dann Hegels Kritik gefunden. ${ }^{89}$

\section{Schlußfolgerungen}

Der historische Durchgang, exemplarisch wie er nur sein konnte, ist damit beendet. Abschließend sei folgende Frage gestellt: Lassen sich aus dem Dargelegten über die immer lehrreiche Kenntnis der historischen Vielfalt möglicher Ansätze und Inhaltsbestimmungen hinaus allgemeine Kenntnisse im Blick auf den Gemeinwohlbegriff, seine semantische Struktur und Aussagekraft, gewinnen? Ich meine, ja.

Die Vielfalt, die uns begegnet ist, läßt sich nicht als kontinuierlicher geschichtlicher Entwicklungsgang interpretieren, so als ob der Gemeinwohlbegriff oder auch Gemeinwohlvorstellungen einer eigenen, ihnen eingepflanzten Teleologie folgten, die sich in der Geschichte entfaltet und schließlich über verschiedene Stufen hinweg zum ,wahren Gemeinwohlbegriff führt. Dafür sind die Ansätze und Inhaltsbestimmungen zu unterschiedlich und heterogen. Eine innere Dialektik des Begriffs ist nicht aufweisbar.

Auf der anderen Seite läßt sich - auch das zeigt die gegebene Darstellung - der Gemeinwohlbegriff nicht auf eine beliebig auffüllbare Leerformel reduzieren, die ohne sachlichen Ertrag bleibt. Sieht man genauer hin, so zeigt sich nämlich ungeachtet von Unterschieden und Heterogenität eine durchgehende Gemeinsamkeit: Gemeinwohlbegriff und Gemeinwohlvorstellungen sind abhängig und inhaltlich geprägt von den Auffassungen über das Um-willen und die Legitimation der politischen Gemeinschaft. Das Gemeinwohl fungiert als Zielbegriff, um das, was Aufgabe der politischen Gemeinschaft bzw. des Staates - oder auch einer Weltgemeinschaft - ist und ihre gute Ordnung ausmacht, umzusetzen und zu realisieren. Insofern ist der Gemeinwohlbegriff stets ein staatsphilosophisch bzw. staatstheoretisch affizierter Begriff und von dieser Grundlage nicht ablösbar; er ist deshalb auch ein - wie auch immer - gehaltvoller, nicht gehaltloser Begriff.

Eine weitere Gemeinsamkeit tritt hinzu. Gemeinwohlbegriff und Gemeinwohlvorstellungen weisen durchweg sowohl inhaltlich-normative wie auch institutionell-organisatorische Momente auf. Je nach dem Ansatz, von dem aus argumentiert wird, und der

${ }^{88} \mathrm{Vgl}$. ebd.

${ }^{89} \mathrm{Vgl}$. Hegel 1959a, S. 329 ff. (§ 258 Anm.). 
Wahrnehmungsperspektive im Blick auf die umgebende Wirklichkeit stehen mal die einen, mal die anderen Momente im Vordergrund. Dabei läßt sich sagen, daß die ethisch-normative Seite des Gemeinwohlbegriffs, historisch gesehen, in besonderer Weise von den christlichen Rechts- und Staatsphilosophen ausgearbeitet und zur Geltung gebracht wird. Das hängt mit den Grundlagen und der Eigenart der christlichen Weltsicht zusammen.

Lassen sich aus diesen Resultaten nun auch Folgerungen für die Verwendbarkeit und Tragfähigkeit des Gemeinwohlbegriffs in juristischen Zusammenhängen und als Rechtsbegriff gewinnen? - Diese Frage kann und sollte vor allem den Juristen beschäftigen, aber nicht den Juristen allein. Für Ihre Beantwortung möchte ich einige Hinweise geben - auch als Anregung für die Diskussion.

Der Gemeinwohlbegriff läßt sich im Rahmen der Rechtsordnung nicht als ein eindeutig fixierter rechtlicher Begriff verwenden, denn er ist ein solcher Begriff nicht. Rechtsdogmatisch gesehen, handelt es sich bei ihm um einen offenen normativen Begriff, ${ }^{90}$ der ausfüllungsbedürftig ist und damit auch ideologischer Okkupation zugänglich. Daß dieser Begriff indes nicht nur ausfüllungsbedürftig, sondern auch ausfülllungsfähig ist, ohne der Beliebigkeit oder ideologischer Beschlagnahme anheimfallen zu müssen, ergibt sich daraus, daß er, wie wir gesehen haben, aus sich heraus in einem staatsphilosophischen oder jedenfalls staatstheoretischen Bezug steht, der seine sachlogische Struktur prägt. Deshalb ist es möglich, aber auch geboten, seine Ausfüllung an diesem Bezug zur Staatslegitimation und den - womöglich auch verfassungsrechtlich fixierten - Zielen und Zwecken, um deretwillen der Staat besteht, zu orientieren und zu messen. Auf diese Weise wird der Gemeinwohlbegriff zu einem sogenannten Scharnierbegriff, der auf die jeweils anerkannte Staatslegitimation einschließlich der Staatsziele und Staatsaufgaben zurückverweist und diese - auch in ihrer sich entwickelnden und verändernden Gestalt - auf die Verfassungsebene transponiert und in die Rechtsordnung hinein umsetzt.

So angewendet und gehandhabt, ist der Gemeinwohlbegriff ungeachtet seiner Offenheit von Beliebigkeit oder Leerformelhaftigkeit weit entfernt. Er fungiert dann - und kann das auch - als tragfähige Brücke zu vor-positiven legitimatorischen Gehalten der Verfassungs- und Rechtsordnung, die er innerhalb dieser Ordnung lebendig erhält. ${ }^{91}$

90 Engisch 1996, S. 162-166.

9) In der Diskussion hat Gunnar F. Schuppert auf drei Ebenen des Gemeinwohlbegriffs als Rechtsbegriff hingewiesen: die substantielle Dimension - bezogen auf materielle Gehalte, die Verfahrensformen zur Ermittlung und Festlegung von Gemeinwohlgehalten und die Ausdifferenzierung des Begriffs in einzelne Gemeinwohlbelange, die jeweils zu berücksichtigen und ggf. miteinander auszugleichen sind. 
Literatur:

Aristoteles $\left(1972^{3}\right)$, Nikomachische Ethik, auf d. Grundlage d. Übers. v. E. Rolfes hg. v. G. Bien, Hamburg.

Aristoteles $\left(1981^{4}\right)$, Politik, übers. u. m. Anm. versehen v. E. Rolfes, Hamburg.

Cicero (1994), De legibus, lat. u. dt., hg. u. übers. v. R. Nickel, München.

Cicero $\left(1987^{3}\right)$, De officiis, lat. u. dt., hg. u. übers. v. K. Büchner, München.

Cicero $\left(1993^{5}\right)$, De republica, lat. u. dt., hg. u. übers. v. K. Büchner, München.

Deckers, D. (1991), Gerechtigkeit und Recht. Eine historisch-kritische Untersuchung der Gerechtigkeitslehre des Francisco de Vitoria (1483-1546), Freiburg i. Ü./Freiburg i. B.

Depenheuer, O. (1988), Der Wortlaut als Grenze, Heidelberg.

Engisch, K. (1996 $\left.{ }^{9}\right)$, Einführung in das juristische Denken, Stuttgart.

Gigon, O. (1976), Gegenwärtigkeit und Utopie. Eine Interpretation von Platons „Staat“, Bd. 1, Zürich/München.

Girardet, K. M. (1983), Die Ordnung der Welt. Ein Beitrag zur philosophischen und politischen Interpretation von Ciceros Schrift De legibus, Wiesbaden.

Graeser, A. (19932), Philosophie der Antike 2, München.

Guthrie, W. C. K. (1975), A History of Greek Philosophy, vol. 4, Cambridge.

Hegel, G. W. F. (1959a), Grundlinien der Philosophie des Rechts, Sämtliche Werke, hg. v. H. Glockner, Bd. 7, Stuttgart-Bad Canstatt.

Hegel, G. W. F. (1959b), Vorlesung über die Geschichte der Philosophie, Sämtliche Werke, hg. v. H. Glockner, Bd. 18, Stuttgart-Bad Canstatt.

Hobbes, T. (1983), De cive. The latin version, a critical edition by H. Warrender, Oxford.

Hobbes, T. (1991), Leviathan, ed. by R. Tuck, Cambridge.

Kamp, A. (1985), Die politische Philosophie des Aristoteles und ihre metaphysischen Grundlagen, Freiburg.

Kant, I. (1977a), Idee zu einer allgemeinen Geschichte in weltbürgerlicher Absicht, in: Werkausgabe, hg. v. W. Weischedel, Bd. 11, Frankfurt/M., S. 30-50.

Kant, I. (1977b), Die Metaphysik der Sitten, Werkausgabe, hg. v. W. Weischedel, Bd. 8, Frankfurt/M.

Kant, I. (1977c), Über den Gemeinspruch: Das mag in der Theorie richtig sein, taugt aber nicht für die Praxis, in: Werkausgabe, hg. v. W. Weischedel, Bd. 11, Frankfurt/M., S. 125-172.

Koselleck, R. (1992 ${ }^{4}$ ), Einleitung, in: Geschichtliche Grundbegriffe, hg. v. O. Brunner, W. Conze und R. Koselleck, Bd. 1, Stuttgart, S. XXII-XXIV.

Kroeschell, K. (1983), Verfassungsgeschichte und Rechtsgeschichte des Mittelalters, in: Gegenstand und Begriffe der Verfassungsgeschichtsschreibung, Der Staat, Beiheft 6, Berlin, S. 66-74.

Las Casas, B. de (1992), Obras Completas 10: Tratados de 1552, Madrid.

Las Casas, B. de (1990), Obras completas 12: De regia potestate, Madrid.

Las Casas, B. de (1996), Werkauswahl, hg. v. M. Delgado, Bd. 3/1: Sozialethische und staatsrechtliche Schriften, Paderborn.

Las Casas, B. de (1997), Werkauswahl, hg. v. M. Delgado, Bd. 3/2: Sozialethische und staatsrechtliche Schriften, Paderborn.

Locke, J. (1988), Two Treatises of Government, ed. with an Introduction and notes by P. Laslett, Cambridge.

Macpherson, C. B. (1973), Die politische Theorie des Besitzindividualismus, Frankfurt/M.

Meyer, E. $\left(1962^{2}\right)$, Römischer Staat und Staatsgedanke, Zürich. 
Meyer, H. (1964²), Thomas v. Aquin, Paderborn.

Platon, (1976), Der Staat, eingel., übers. u. erkl. v. K. Vretska, Stuttgart.

Platon (1993), Kriton, in: Platon, Sämtliche Dialoge, hg. v. O. Apelt, Bd. 1, Hamburg.

Schilling, K. $\left(1966^{2}\right)$, Geschichte der sozialen Ideen, Stuttgart.

Schmitt, C. (1958), Nehmen - Teilen - Weiden, in: ders., Verfassungsrechtliche Aufsätze, Berlin, S. 489-504.

Schütrumpf, E. (1991), Aristoteles. Politik II und III, übers. u. erl. v. E. Schütrumpf, Darmstadt.

Suárez, F. (1972), De legibus I.9-20: de legis obligatione, edición crítica bilingüe, Corpus Hispanorum de pace Bd. 12, Madrid.

Suárez, F. (1975), De legibus III.1-16: de civili potestate, edición crítica bilingüe, Corpus Hispanorum de pace Bd. 15, Madrid.

Thomas v. Aquin (1979), De Regno, in: Sancti Thomae de Aquino Opera omnia, iussu Leonis XIII P. M. edita, Bd. 42, Rom.

Thomas v. Aquin (1918-1930), Summa contra gentiles cum commentariis Ferrariensis, in: Sancti Thomae de Aquino Opera omnia, iussu Leonis XIII P. M. edita, Bde. 13-15, Rom.

Thomas v. Aquin (1888-1906), Summa theologiae cum Supplemento et commentariis Caietani, in: Sancti Thomae de Aquino Opera omnia, iussu Leonis Xlll P. M. edita, Bde. 4-12, Rom.

Trevelyan, G. M. (1948), Kultur- und Sozialgeschichte Englands, Hamburg. 